\title{
Lista das espécies de Calliphoridae (Diptera, Oestroidea) do Estado do Mato Grosso do Sul, Brasil
}

\author{
Cecília Kosmann', Anna Carolina Prestes' ${ }^{1}$ Karla Pessôa Tepedino' , Ana Carolina Franco', \\ Cristiane V. A. Pujol-Luz² \& José Roberto Pujol-Luz',2
}

\begin{abstract}
1. Universidade de Brasília, Instituto de Ciências Biológicas, Departamento de Zoologia, 70910-900 Brasília, DF. (jrpujol@unb.br) 2. Universidade Católica de Brasília, Laboratório de Zoologia, QS 7 lote 1, BI. M/ s. 331, 72030-170 Taguatinga, DF
\end{abstract}

Recebido 8 dezembro 2016

Aceito 6 fevereiro 2017

DOI: $10.1590 / 1678-4766 e 2017140$

ABSTRACT. Checklist of the Calliphoridae (Diptera, Oestroidea) from Mato Grosso do Sul, Brazil state. In this paper it is provided a checklist of the Calliphoridae species registered in the state of Mato Grosso do Sul. Calliphoridae is represented by 13 species distributed in seven genera: Chloroprocta Wulp (1), Chrysomya Robineau-Desvoidy (3), Cochliomyia Townsend (2), Hemilucilia Brauer (2), Paralucilia Brauer \& Bergenstamm (1), Lucilia Robineau-Desvoidy (2), and Mesembrinella Giglio-Tos (2).

KEYWORDS. Biodiversity, blow flies, Cerrado, Pantanal, Biota-MS Program.

RESUMO. Neste trabalho é apresentada uma lista de espécies de Calliphoridae registradas no estado do Mato Grosso do Sul. Calliphoridae é representada por 13 espécies distribuídas em sete gêneros: Chloroprocta Wulp (1), Chrysomya Robineau-Desvoidy (3), Cochliomyia Townsend (2), Hemilucilia Brauer (2), Paralucilia Brauer \& Bergenstamm (1), Lucilia Robineau-Desvoidy (2) e Mesembrinella Giglio-Tos (2).

PALAVRAS-CHAVE. Biodiversidade, Cerrado, moscas-varejeiras, Pantanal, Programa Biota-MS

Os membros de Calliphoridae (Calyptratae, Oestroidea) são moscas de tamanho mediano e coloração metálica, comumente conhecidos como moscas-varejeiras. Possuem distribuição mundial, com mais de 1.000 espécies descritas em aproximadamente 150 gêneros (SHEWELL, 1987; VARGAS \& WoOD, 2010). Uma vez que trata-se de uma família de grande impacto na sociedade humana, existe a necessidade de estabilidade no grupo, levando a uma classificação conservativa (SHewell, 1987). Atualmente a família é dividida em 12 subfamílias: Auchmeromyiinae, Bengaliinae, Phumosiinae, Mesembrinellinae, Helicoboscinae, Toxotarsinae, Calliphorinae, Chrysomyinae, Luciliinae, Melanomyinae, Polleniinae e Rhiniinae (Rognes, 1997). Na Região Neotropical há registro de sete subfamílias, enquanto que no Brasil este número é de cinco.

Dentre todas as famílias do grupo Oestroidea, Calliphoridae é a única que não possui seu monofiletismo confirmado (PAPE, 1992; Rognes, 1997). Todavia, com exceção de Mesembrinellinae, as espécies do Novo Mundo parecem formar um grupo monofilético (VARGAS \& WoOD, 2010). O fóssil mais antigo atribuído à família data do Cretáceo superior (70 milhões de anos), tendo sido descrito por MCALPINE (1970) a partir de quatro pupários que ele atribuiu à nova espécie Cretaphormia fowleri. Este foi o primeiro registro pré-terciário de um oestróideo (RoGNES, 1997).
Os adultos e imaturos de Calliphoridae possuem hábitos diversos, sendo o mais comum o desenvolvimento em carcaças de animais (VARGAS \& WooD, 2010). Este hábito torna as espécies desta família importantes na cadeia de reciclagem de nutrientes (BYRD \& CASTNER, 2001) e serve como ferramenta para estimativa do intervalo pósmorte (IPM) em investigações criminais (BENECKE, 2001; Amendt et al., 2007; Pujol-Luz et al., 2008). Adultos são ainda encontrados alimentando-se em fezes, néctar e secreções açucaradas, bem como polinizando diversas espécies de plantas (JiRón \& HEDSTRöM, 1985). Além do desenvolvimento em carcaças, as larvas podem utilizar fezes e material vegetal em decomposição como substrato de criação, bem como ser parasitas de répteis (Koskela et al., 1974), gastrópodes e anelídeos (RoGNES, 1991). Espécies de três gêneros são conhecidas por alimentarem-se de sangue humano e de outros animais (VARGAS \& WoOD, 2010). A biologia das espécies brasileiras é bem estudada, com exceção da sufamília Mesembrinellinae.

O elevado número de estudos relacionados à família deve-se, em grande parte, à importância médico-veterinária apresentada por muitas de suas espécies, reportadas como causadoras de miíases (ZUMPT, 1965; GUIMARÃES et al., 1983; HaLl \& Wall, 1995) e vetores mecânicos de patógenos aos animais e ao homem (GREENBERG, 1971). Os danos 
econômicos na criação de gado devido às miíases são extensivos, levando inclusive a medidas para erradicação total de algumas espécies (e.g. Cochliomyia macellaria Fabricius nos Estados Unidos da América, México, Belize e Costa Rica).

A fauna brasileira de califorídeos é consideravelmente bem conhecida se comparada à de outros grupos. Estudos relacionados à associação das espécies com o ambiente urbano - sinantropia -, bem como relatos de miíases causadas por califorídeos são frequentes (LINHARES, 1981; D'ALMEIDA \& Lopes, 1982; Guimarães \& PAPAVERO, 1999; ThysSEn $e t$ al., 2012).

Para o estado do Mato Grosso do Sul, poucos são os trabalhos com califorídeos (CAMPOS \& BARROS, 1995), sendo a maioria deles realizados em locais associados à criação de gado (Gomes et al., 2000; CorRÊA et al., 2010; Koller et al., 2011).

\section{MATERIAL E MÉTODOS}

A lista das espécies de califorídeos que ocorrem no Estado do Mato Grosso do Sul foi elaborada com base na literatura disponível entre os anos 1960 e 2013. Destacam-se Mello (1961, 1962, 1965, 1967, 1968, 1969a,b, 1972a,b, 1974, 1978, 1996, 2003), JAMES (1970), DEAR (1979, 1985), Guimarães (1977), Kosmann et al. (2013), Lopes \& Albuquerque (1982), Mariluis (1979, 1980, 1983), Mariluis \& Peris (1984), Peris \& Mariluis (1984), Shannon (1926) e Whitworth (2006, 2010, 2012). Os catálogos de Stone et al. (1965), Kurahashi (1989) e PonT (1980) também foram consultados.

Neste trabalho consideramos Mesembrinellinae como uma subfamília baseada nas contribuições de RoGNES (1986) e TOMA \& CARVAlHo (1995), em detrimento a GuIMARÃES (1977). As espécies exóticas foram assinaladas com $(* \mathrm{E})$.

\section{RESULTADOS E DISCUSSÃO}

Existem registros de sete gêneros e 13 espécies de califorídeos no Mato Grosso do Sul, distribuídas em três subfamílias: Chrysomyinae (cinco gêneros e nove espécies), Luciliinae (um gênero e duas espécies) e Mesembrinellinae (um gênero e duas espécies).

Apesar de haver o registro de 43 espécies no Brasil, apenas 13 são encontradas no estado do Mato Grosso do Sul. Este número reduzido deve-se, em parte, ao fato de que os esforços de coleta e estudos da família ficam restritos ao âmbito agropecuário e veterinário, visto ser esta uma das bases da economia local. A grande importância médicaveterinária que as espécies apresentam acaba por direcionar os estudos, levando a lacunas no conhecimento da fauna do estado.

\section{Lista de espécies de Calliphoridae do Estado do Mato Grosso do Sul}

\section{CHRYSOMYINAE Chloroprocta Wulp, 1896}

idioidea (Robineau-Desvoidy, 1830). Localidade-tipo: Brasil. Distribuição: Argentina, Bahamas, Brasil, Colômbia, Costa Rica, Cuba, El Salvador, Equador, EUA, Guatemala, Guiana, Guiana Francesa, México, Nicarágua, Panamá, Paraguai, Peru, Trindade e Tobago, Venezuela.

\section{Chrysomya Robineau-Desvoidy, 1830}

albiceps (Wiedemann, 1819). (*E) Localidade-tipo: África do Sul, Cabo da Boa Esperança. Distribuição na Região Neotropical: Argentina, Bolívia, Brasil, Colômbia, Dominica, Guatemala, Nicarágua, Paraguai, Peru, Porto Rico, Uruguai, Venezuela.

megacephala (Fabricius, 1794). (*E) Localidade-tipo: "Guinea", [error =? "Ex. Ind. Or."]. Distribuição na Região Neotropical: Argentina, Brasil, Colômbia, Dominica, Grandes Antilhas, Jamaica, Porto Rico, Peru, República Dominicana.

putoria (Wiedemann, 1818). (*E) Localidade-tipo: Serra Leoa. Distribuição na Região Neotropical: Argentina, Bolívia, Brasil, Colômbia, Panamá, Paraguai, Peru.

\section{Cochliomyia Townsend, 1915}

hominivorax (Coquerel, 1858). Localidade-tipo: "Guiana”. Distribuição: Argentina, Brasil, Chile, Colômbia, Costa Rica, Cuba, Grandes Antilhas, Guatemala, Guiana Francesa, Jamaica, México, Nicarágua, Panamá, Peru, Porto Rico, República Dominicana, Trindade e Tobago, Uruguai.

macellaria (Fabricius, 1775). Localidade-tipo: "West Indies". Distribuição: Argentina, Bahamas, Belize, Bermudas, Bolívia, Brasil, Caribe, Chile, Colômbia, Costa Rica, Cuba, Equador, Grandes Antilhas, Guatemala, Guiana, Honduras, Jamaica, México, Nicarágua, Panamá, Paraguai, Peru, República Dominicana, Trindade e Tobago, Uruguai, Venezuela.

\section{Hemilucilia Brauer, 1895}

segmentaria (Fabricius, 1805). Localidade-tipo: "América do Sul". Distribuição: Argentina, Bolívia, Brasil, Chile, Colômbia, Costa Rica, El Salvador, Equador, Guatemala, Guiana, México, Panamá, Paraguai, Peru, Trindade e Tobago. 
semidiaphana (Rondani, 1850). Localidade-tipo: Argentina, Brasil, São Paulo, Ilha São Sebastião. Distribuição: Bolívia, Brasil, Colômbia, Costa Rica, Equador, Guatemala, Guiana, Panamá, Paraguai, Peru, Trindade e Tobago, Venezuela.

\section{Paralucilia Brauer \& Bergenstamm, 1891}

pseudolyrcea (Mello, 1969). Localidade-tipo: Brasil, Santa Catarina, Nova Teutônia. Distribuição: Argentina, Bolívia, Brasil, Colômbia, Paraguai.

\section{LUCILIINAE}

\section{Lucilia Robineau-Desvoidy, 1830}

cuprina (Wiedemann, 1830). (*E) Localidade-tipo: "China”. Distribuição na Região Neotropical: Argentina, Bermuda, Brasil, Colômbia, Cuba, Haiti, Ilhas Virgens, Jamaica, Peru, Porto Rico, Trindade, Uruguai, Venezuela.

eximia (Wiedemann, 1819). Localidade-tipo: "Brasil”.

Distribuição: Argentina, Barbados, Brasil, Chile, Colômbia, Costa Rica, Dominica, Equador, Granada, Guadalupe, Guatemala, Ilhas Virgens, México, Nicarágua, Peru, Porto Rico, República Dominicana, São Vicente, Trindade, Venezuela.

\section{MESEMBRINELLINAE Mesembrinella Giglio-Tos, 1893}

batesi Aldrich, 1922. Localidade-tipo: Brasil, Amazonas. Distribuição: Brasil, Colômbia, Peru.

bicolor (Fabricius, 1805). Localidade-tipo: "America meridionalis", Copenhagen. Distribuição na Região Neotropical: México ao Panamá. América do Sul, exceto Chile e sul da Argentina.

Agradecimentos. Os autores agradecem a Fundação de Apoio ao Desenvolvimento do Ensino, Ciências e Tecnologia do Estado de Mato Grosso do Sul (Fundect) e a Superintendência de Ciências e Tecnologia do Estado de Mato Grosso do Sul (Sucitec/MS) pelo convite de participação neste fascículo especial da Iheringia, Série Zoologia e o suporte financeiro para sua publicação; e às seguintes agências de fomento à pesquisa: Conselho Nacional de Desenvolvimento Científico e Tecnológico (CNPq); Fundação de Amparo à Pesquisa do Estado de São Paulo (FAPESP); Fundação de Apoio à Pesquisa do Distrito Federal (FAPDF); Coordenação de Aperfeiçoamento de Pessoal de Nível Superior (CAPES); Financiadora de Estudos e Projetos (FINEP), Pró-Reitoria de Pesquisa e Pós-Graduação da Universidade Católica de Brasília (UCB)

\section{REFERÊNCIAS BIBLIOGRÁFICAS}

Amendt, J.; Campobasso, C. P.; Gaudry, E.; Reiter, C.; Leblanc, H. N. \& HaLl, M. J. R. 2007. Best practice in forensic entomology - Standards and guidelines. International Journal of Legal Medicine 121:90-104

BeneCKe, M. 2001. A brief history of forensic entomology. Forensic Science International 120:2-14

Byrd, J. H. \& CASTnER, J. L. 2001. Insects of forensic importance. In: Byrd, J. H. \& CAStner, J. L. eds. Forensic Entomology. The utility of arthropods in legal investigations. Florida, CRC Press LLC, p. 43-80.

Campos, C. F. M. \& Barros, A. T. M. 1995. Dípteros muscóides da área urbana de Corumbá, Mato Grosso do Sul, Brasil. Revista Brasileira de Entomologia 55:347-350.
CorrêA, E. C.; Koller, W. W. \& Barros, A. T. M. 2010. Abundância relativa e sazonalidade de espécies de Chrysomya (Diptera: Calliphoridae) no Pantanal Sul-Mato-Grossense. Revista Brasileira de Parasitologia Veterinária 19:85-88

D’Almeida, J. M. \& Lopes, H. DE. S. 1983. Sinantropia de dípteros caliptrados (Calliphoridae) no Estado do Rio de Janeiro. Arquivos da Universidade Federal Rural do Rio de Janeiro 6:31-38.

Dear, J. P. 1979. A revision of the Toxotarsinae (Diptera: Calliphoridae). Papéis Avulsos de Zoologia 32:145-182.

DeAR, J. P. 1985. A revision of the new world Chrysomyini (Diptera: Calliphoridae). Revista Brasileira de Zoologia 3:109-169.

Gomes, A.; Koller, W. W. \& Barros, A. T. M. 2000. Sazonalidade da mosca-varejeira, Cochliomyia macellaria (Diptera: Calliphoridae), na região dos Cerrados, Campo Grande, MS. Revista Brasileira de Parasitologia Veterinária 9:125-128.

Greenberg, B. 1971. Flies and disease. Ecology, Classification and Biotic Associations. New Jersey, Princeton University Press. vol. 1. 856p.

Guimarães, J. H. 1977. A Systematic revision of the Mesembrinellidae, stat. nov. (Diptera, Cyclorrahapha). Arquivos de Zoologia 29:1-109.

Guimarães, J. H. \& PAPAVERo, N. 1999. Myiasis in man and animals in the Neotropical Region, bibliographic database. São Paulo, Plêiades; FAPESP. 308p.

Guimarães, J. H.; Papavero, N. \& Prado, A. P. 1983. As miíases na região Neotropical (Identificação, biologia, bibliografia). Revista Brasileira de Zoologia 1:239-416.

Hall, M. \& WALL, R. 1995. Myiasis of humans and domestic animals. Advanced in Parasitology 35:257-334.

James, M. T. 1970. Family Calliphoridae. In: PAPAVero, N. ed. A catalogue of the Diptera of Americas south of the United States. São Paulo, Departamento de Zoologia, Secretaria de Agricultura. vol. 102, p.1-28.

Jirón, L. F. \& Hedström, I. 1985. Pollination ecology of mango (Mangifera indica L.) (Anacardiaceae) in the Neotropic region. Turrialba 35:269277.

Koller, W. W.; Barros, A. T. M. \& Correa, E .C. 2011. Abundance and seasonality of Cochliomyia macellaria (Diptera: Calliphoridaea) in Southern Pantanal, Brazil. Revista Brasileira de Parasitologia Veterinária 20:27-30.

Koskela P.; IтÄmies, J. \& Pasane, S. 1974. Lucilia bufonivora Moniez (Dipt., Calliphoridae), a lethal parasite in Rana temporaria L. (Anura). Annales Zoologici Fennici 11:105-106.

Kosmann, C.; Mello, R. P.; Harterreiten-Souza E. S. \& Pujol-Luz, J. R. 2013. A List of Current Valid Blow Fly Names (Diptera: Calliphoridae) in the Americas South of Mexico with Key to the Brazilian Species. EntomoBrasilis 6:74-85.

Kurahashi, H. 1989. Family Calliphoridae. In: Evenhuis, N. L. ed. Catalog of the Diptera of the Australasian and Oceanian Region. Honolulu, Special Publications of Bernice P., Bishop Museum, p. 702-718.

Linhares, A. X. 1981. Synanthropy of Calliphoridae and Sarcophagidae (Diptera) in the city of Campinas, São Paulo, Brazil. Revista Brasileira de Entomologia. 25:189-215.

Lopes, H. De S. \& Albuquerque, D. De O. 1982. Notes on Neotropical Calliphoridae (Diptera). Revista Brasileira de Biologia 42:63-69.

Mariluis, J. C. 1979. Notas sobre algunas especies del genero Hemilucilia Brauer, 1895 (Diptera: Calliphoridae). Physis 38:68; 86; 97.

Mariluis, J. C. 1980. Contribuition al conocimiento del genero Hemilucilia Brauer (Calliphoridae, Chrysomyinae, Chrysomyiini). Revista de la Sociedad Entomológica Argentina 39:83-87.

Mariluis, J. C. 1983. Presencia del género Chrysomya Robineau-Desvoidy, 1830 en la région Neotropical (Calliphoridae, Chrysomyinae, Hemiluciliini). Revista de la Sociedad Entomológica Argentina 42:141-142.

Mariluis, J. C. \& Peris, S. V. 1984. Datos para una sinopsis de los Calliphoridae Neotropicales. Eos 60:67-86.

McAlpine, J. F. 1970. First record of calyptratae flies in the Mesozoic era (Diptera: Calliphoridae). The Canadian Entomologist 102:342-346.

Mello, R. P. DE. 1961. Contribuição ao estudo do gênero Phaenicia (R. D., 1830) (Diptera, Calliphoridae). Memórias do Instituto Oswaldo Cruz 59:259-278.

Mello, R. P. DE. 1962. Contribuição ao estudo do gênero Calliphora R.D., 1830 (Diptera, Calliphoridae). Memórias do Instituto Oswaldo Cruz 60:263-274. 
Mello, R. P. DE. 1965. Contribuição ao estudo do gênero Callitrogopsis Townsend (Diptera, Calliphoridae). Studia Entomologica 8:435-440.

Mello, R. P. DE. 1967. Contribuição ao estudo dos Mesembrinellinae sulamericanos (Calliphoridae). Studia Entomologica 10:1-80.

Mello, R. P. DE. 1968. Contribuição ao estudo do gênero "Paralucilia" Brauer \& Bergenstamm, 1891 (Calliphoridae, Diptera). Revista Brasileira de Biologia 28:177-192.

Mello, R. P. DE. 1969a. Notes on Laneella brunnipes (Surcouf, 1919) (Diptera, Calliphoridae). Revista Brasileira de Biologia 29:243-247.

Mello, R. P. DE. 1969b. Contribuição ao estudo do gênero Myiolucilia Hall (Diptera, Calliphoridae). Studia Entomologica 12:297-316.

Mello, R. P. DE. 1972a. Contribuição ao estudo do gênero Sarconesia Bigot, 1857 (Diptera, Calliphoridae). Revista Brasileira de Biologia 32:533-537.

Mello, R. P. DE. 1972b. Revisão das espécies do gênero Hemilucilia Brauer, 1895 (Diptera, Calliphoridae). Revista Brasileira de Biologia 32:539-554.

Mello, R. P. DE. 1974. Nova espécie do gênero Calliphora RobineauDesvoidy, 1830. (Diptera, Calliphoridae). Arquivos da Universidade Federal Rural do Rio de Janeiro 4:59-63.

Mello, R. P. DE. 1978. Contribuição ao conhecimento do gênero Roraimomusca Townsend, 1935 (Diptera, Calliphoridae). Revista Brasileira de Biologia 32:895-898.

Mello, R. P. DE. 1996. Revisão das espécies sul americanas de Paralucilia Brauer \& Bergenstamm (Diptera: Calliphoridae). Entomología y Vectores 3:137-143.

Mello, R. P. DE. 2003. Chave para a identificação das formas adultas das espécies da família Calliphoridae (Diptera, Brachycera, Cyclorrhapha) encontradas no Brasil. Entomología y Vectores 10:255-268.

PAPE, T. 1992. Phylogeny of the Tachinidae family-group (Diptera: Calyptratae). Tijdschrift voor Entomologie 135:43-86.

Peris, S. V. \& Mariluis, J. C. 1984. Notes on Mesembrinellidae. Eos 60:251-265.

Pont, A. C. 1980. Family Calliphoridae. In: Crosskey, R. M. ed. Catalogue of the Diptera of the Afrotropical Region. London, British Museum (Natural History), p. 779-800.

Pujol-Luz, J. R.; Arantes, L. C. \& Constantino, R. 2008. Cem anos da entomologia forense no Brasil. Revista Brasileira de Entomologia 52:485-492.
Rognes, K. 1986. The systematic position of the genus Helicobosca Bezzi with a discussion of the monophyly of the calyptratae family's Calliphoridae, Rhinophoridae, Sarcophagidae and Tachinidae (Diptera). Entomologica Scandinavica 17:75-92.

Rognes, K. 1991. Blowflies (Diptera, Calliphoridae) of Fennoscandia and Denmark. Fauna Entomologica Scandinavica 24:1-272.

Rognes, K. 1997. The Calliphoridae (blowflies) (Diptera: Oestroidea) are not a monophyletic group. Cladistics 13:27-66.

Shannon, R. C. 1926. Synopsis of the American Calliphoridae (Diptera). Proceedings of the Entomological Society of Washington 28:115-139.

Shewell, G. E. 1987. Calliphoridae. In: (McAlpine, J. F.; Peterson, B. V.; Shewell, G. E.; Teskey, H. J.; Vockeroth, J. R. \& Wood, D. M. eds. Manual of Neartic Diptera. Ottawa, Monograph/Agriculture Canada, vol. 2., p. 1113-1145.

Stone, A.; Sabrosky, C. W.; Wirth, W. W.; Foote, R. H. \& Coulson, J. R. 1965. A catalog of the Diptera of America north of Mexico. Washington, Agriculture Handbook, United States Department of Agriculture. 1696p.

Thyssen, P. J.; Nassu, M. P.; Costella, A. M. U. \& Costella, M. L. 2012. Record of oral myiasis by Cochliomyia hominivorax (Diptera: Calliphoridae): case evidencing negligence in the treatment of incapable. Parasitology Research 111:957-959.

Toma, R. \& Carvalho, C. J. B. DE. 1995. Estudo filogenético de Mesembrinellinae com ênfase no gênero Eumesembrinella Townsend (Diptera, Calliphoridae). Revista Brasileira de Zoologia 12:127-144.

VArgas, J. \& Wood, D. M. 2010. Calliphoridae. In: Brown, B. V.; Borkent, A.; Cumming, J. M.; Wood, D. M.; Woodley, N. E. \& Zumbado, M. A. eds. Manual of Central American Diptera. Boca Raton, NCR Research Press, vol. 2., p.1297-1304.

WhitworTH, T. 2006. Keys to the genera and species of blow flies (Diptera: Calliphoridae) of America north of Mexico. Proceedings of the Entomological Society of Washington 108: 689-725.

WhitworTh, T. 2010. Keys to the genera and species of blow flies (Diptera: Calliphoridae) of the West Indies and description of a new species of Lucilia Robineau-Desvoidy. Zootaxa 2663:1-35.

WhitworTH, T. 2012. Identification of Neotropical blow flies of the genus Calliphora Robineau-Desvoidy (Diptera: Calliphoridae) with the description of a new species. Zootaxa 3209:1-27.

ZuMPT, F. 1965. Myiasis in man and animals in the Old World.A textbook for physicians, veterinarians and zoologists. London, Butterworths. 267p. 